\title{
PAUTAS DE MANEJO DE LAS MALEZAS PARA INCREMENTAR LAS POBLACIONES DE INSECTOS BENÉFICOS EN EL CULTIVO DE PALMA ACEITERA (Elaeis guineensis Jacquin) ${ }^{1}$
}

\author{
Ramón Mexzón ${ }^{2}$
}

\begin{abstract}
RESUMEN
Algunas pautas de manejo de las malezas para incrementar los insectos benéficos en el cultivo de palma aceitera (Elaeis guineensis Jacquin). Un total de 61 especies de malezas son informadas en el cultivo de palma aceitera en las que se hospeda o alimenta parte de la entomofauna. Las plantas más visitadas por los insectos fueron, en orden descendente, especies de Asteraceae, Euphorbiaceae, Leguminosae y Malvaceae. De estas especies 23 eran anuales y 38 eran perennes. Una mayoría de las especies crecían en las siembras nuevas de palma sin la cobertura de kudzú, también en las orillas de caminos, de canales de drenaje y en espacios vacantes de la plantación adulta o vieja. Algunas plantas perennes y con glándulas extraflorales situadas en las plantaciones adultas y viejas fueron muy atractivas para la entomofauna. El incremento de las poblaciones de artrópodos (entre ellos los insectos benéfi$\cos$ ) se podría obtener a través de un manejo conservativo de la vegetación, se sugieren cinco estratégias. Se señala la necesidad de apoyar grandes cambios en la vegetación, con base en estudios ecológicos; para evitar favorecer insectos plaga o vectores de organismos patógenos y de evaluar el efecto de estas medidas sobre la salud y rendimiento del cultivo.
\end{abstract}

\begin{abstract}
Guidelines for a weed management that increase the populations of beneficial insects in oil palm (Elaeis guineensis Jacquin). We found that weeds most frequently visited by insects were, in descending order, the species of Asteraceae, Euphorbiaceae, Leguminose and Malvaceae. Of those, 23 were annuals and 38 were perennials. The annual plants flowered during the dry season and the perennial during various periods along the year. Most ofthose species grew in fields with young oil palm trees without the "Kudzu" cover, as well as on roadsides, along canal s and drainage and on clearings inside adult plantations. Some perennial plants with extra-floral glands, growing in fields with adult oil palm trees, proved to be very attractive to insects. The increase in the arthropod population (including beneficial insects), can be obtained through a conservative management of the vegetation. We point out the need to support such changes in the vegetation, based on ecological studies, in order to avoid favoring damaging insects and or pathogenic agents, and also in order to evaluate the effect of such measures over health and yield of the crop.
\end{abstract}

\section{INTRODUCCIÓN}

El ambiente agrícola simplificado propio del mono-cultivo, ha proporcionado muchas oportunidades para que ocurran incrementos poblacionales de artrópodos fitófagos, cuyas necesidades básicas son satisfechas en este tipo de ambiente (Root, 1973; Altieri, 1983; Genty, 1984; Mexzón y Chinchilla, 1992).

En América tropical, en el monocultivo de palma aceitera (Elaeis guineensis Jacquin) se han informado más de 77 especies de artrópodos perjudiciales, de ellas 16 han causado severas pérdidas económicas (Genty, et al., 1978; Reyes y Cruz, 1986). En América Central sólo unas pocas especies de lepidópteros defoliadores han causado daños de importancia económica (Chinchilla, 1992; Mexzón et al., 1992).

Algunos incrementos poblacionales de insectos en palmas se han asociado con la destrucción de sus enemigos naturales por el uso frecuente de insecticidas de amplio espectro o de la vegetación donde se refugian (Syed y Shah, 1976; Hoong y Hoh, 1992; Mexzón, Chinchilla y Salamanca, 1996).

En estas plantaciones la sucesión vegetal se inicia con cerca de 80 especies de malezas en las siembras nuevas; éstas son de rápido crecimiento y producen grandes cantidades de semillas (especies con estrategia R) como algunas amarantáceas, asteráceas y

\footnotetext{
1 Centro de Investigaciones en Protección de Cultivos (CIPROC), Escuela de Fitotecnia, Facultad de Agronomía, Universidad de Costa Rica, San José, Costa Rica.

2 Laboratorio de Entomología Económica, Escuela Fitotecnia, Universidad de Costa Rica.
} 
solanáceas. Esta vegetación por lo general es sustituida por coberturas de leguminosas como el kudzú (Pueraria phaseoloides Bentham) y la pega-pega (Desmodium ovaliofolium Waller) y queda restringida a los espacios vacíos que se producen en el cultivo (especies con estrategia reproductiva $\mathrm{K}$ ).

A los cinco años, el desarrollo de las palmas causa un sombreo desfavorable para la vegetación de producción melífera y es desplazada a las orillas de caminos y de canales de drenajes. En el interior crecen plantas adaptadas a las condiciones de umbría como los helechos, aráceas, platanillas y otras.

Una mayoría de los insectos se alimentan del polen y de las secreciones de glándulas (Leius, 1967; Bombosch, 1973; Syme, 1975) donde obtienen aminoácidos esenciales y carbohidratos que son necesarios en su dieta (Leius, 1967; Braken, 1969; Hagen 1976; Altieri, 1983).

En Costa Rica, en el cultivo de la palmaaceiteraexisten más de 80 especies de malezas asociadas en las que se hospeda y alimenta la entomofauna (Mexzón y Chinchilla, 1990a); de ellas se han documentado 37 especies (Mexzón, 1992). En Colombia y Ecuador, Delvare y Genty (1992) informaron un total de 187 especies de parasitoides en ocho especies de malezas comunes en la palma.

En la actualidad, no se han realizado estudios ecológicos en cultivos perennes para evaluar el impacto del uso de las malezas corno un valioso recurso biológico dentro del agroecosistema, sino que la mayoría de los estudios informan de experimentos de campo en los cuales se prueban nuevos herbicidas en el combate de las mismas.

El presente estudio tiene como objetivo informar acerca de 61 especies de malezas comunes en el cultivo de palma aceitera en la vertiente Pacífica de Costa Rica; y se ofrece una estrategia de manejo de estas plantas con el propósito de incrementar las poblaciones de enemigos naturales de los insectos perjudiciales del cultivo.

\section{MATERIALES Y MÉTODOS}

Un total de 61 especies de malezas fueron estudiados en plantaciones de palma aceitera de la Compañía Palma Tica S.A. situadas en la región sur de la Vertiente del Pacífico de Costa Rica (Cantón de Corredores, Provincia de Puntarenas).

El período de observaciones fue de febrero de 1990 a febrero de 1992 y las condiciones metereológicas de temperatura, humedad relativa y precipitación anual acu- mulada (en ese orden) fueron de 27,3 grados centígrados $(24,5$ a 30,7$), 89 \%$ (79 a 93) y 4.830 mm (DEPT, 1992).

En cada plantación se realizaron observaciones periódicas en las malezas que crecían en las siembras nuevas (<1 a 3,5 años) con o sin kudzú y en las orillas de caminos, de canales de drenaje y espacios sin palmas (vacantes) en las áreas de palmas adultas (5 a 9 años) y viejas (> 10 años). Se realizaron capturas mensuales de los insectos visitantes, los cuales se clasificaron a nivel de familia y de hábito alimentario. Además se anotaron la fenología y la distribución de las plantas en la plantación y algunas interacciones importantes entre los insectos y las plantas.

\section{RESULTADOS Y DISCUSIÓN}

\section{Malezas}

Un total de 61 especies de plantas melíferas, pertenecientes a 21 familias, fueron muy visitadas por los insectos en los sitios de estudio. En orden descendente fueron especies de Asteraceae, Euphorbiaceae, Leguminosae y Malvaceae (Cuadro 1). De estas 23 eran de ciclo anual y 38 de ciclo perenne; 46 crecían en las siembras nuevas, 19 en palmas adultas y 15 en palmas viejas.

La floración de una mayoría de las plantas estudiadas ocurrió durante la estación seca; ésta tiene una duración de 12 a 16 semanas y durante la misma, la cantidad de insectos observada entonces fue notoriamente mayor, debido a la abundancia de polen y de néctar.

En las plantaciones se observó escasez de flora melífera, la cual puede ser interpretada parcialmente por 1la abundancia de gramíneas que son muy competitivos, 2- la chapea y el uso de herbicidas en forma periódica y 3- el incremento paulatino de las condiciones de umbría a medida que crecen las palmas.

En el interior de las plantaciones adultas y viejas algunas plantas herbáceas con glándulas extraflorales fueron muy atractivas para los insectos y en ellas se concentraron gran cantidad de especies de insectos y de individuos por especie. Las plantas más importantes fueron Byttneria aculeata Jacquin, Cassia reticulata L., Crotalaria mucronata L., Melanthera aspera Small, Scleria melaleuca Schlechty Cham.,Senna stenocarpoides (Standley) Britton, Solanum jamaicense Miller y Triunfetta semitriloba L.

En Heliconiaceae y Zingiberaceae algunas especies de escarabajos Chrysomelidaey lepidópteros Limacodidae, Megalopygidae, Nymphalidae y Psychidae fueron comunes (Cuadro 2); de éstas algunas han sido informadas como perjudiciales de la palma en otros países de 
Cuadro 1. Especies vegetales más importantes como huéspedes de insectos en plantaciones de palma aceitera, en el Pacífico Sur de Costa Rica (1990-92).

\begin{tabular}{|c|c|c|c|}
\hline Especie vegetal & Recurso explotado* & Familias insectos** & Observación*** \\
\hline \multicolumn{4}{|l|}{ ANUALES } \\
\hline Blechum brownei & polen & Braconidae & semillas \\
\hline \multirow[t]{2}{*}{ Jusseiu } & & Chrysomelidae & orillas de caminos \\
\hline & & Vespidae & \\
\hline \multirow[t]{2}{*}{ Justicia comata L. } & polen & Braconidae & semillas \\
\hline & & Vespidae & siembras jóvenes \\
\hline \multirow[t]{2}{*}{ Priva aspera H.B.K. } & polen & Chalcididae & semillas \\
\hline & sc.glánd. & Vespidae & orillas de caminos \\
\hline \multicolumn{4}{|l|}{ AMARANTHACEAE } \\
\hline \multirow{4}{*}{ Amarantus spinosus $L$. } & polen & Braconidae & semillas \\
\hline & & Chalcididae & sitios soleados \\
\hline & & Evaniidae & en suelo desnudo \\
\hline & & $\begin{array}{l}\text { Muscidae } \\
\text { otras. }\end{array}$ & \\
\hline \multicolumn{4}{|l|}{ ASTERACEAE } \\
\hline \multirow[t]{3}{*}{ Ageratum conyzoides $L$. } & polen & Braconidae & semillas \\
\hline & & Muscidae & orillas de caminos \\
\hline & & Vespidae & \\
\hline \multirow[t]{3}{*}{ Baltimora recta $L$. } & polen & Apidae & semillas \\
\hline & & Chalcididae & orillas de caminos \\
\hline & & Syrphidae & \\
\hline \multirow[t]{2}{*}{ Bidens pilosa $L$. } & polen & Syrphidae & semillas \\
\hline & & Vespidae & orillas de caminos \\
\hline \multirow[t]{3}{*}{ Eclipta alba $L$. } & polen & Chalcididae & semillas \\
\hline & & Cicadellidae & en suelos desnudos \\
\hline & & Ichneumonidae & \\
\hline \multirow[t]{3}{*}{ Emilia sonchifolia $L$. } & polen & Muscidae & semillas \\
\hline & & Syrphidae & siembras jóvenes \\
\hline & & Vespidae & \\
\hline \multirow[t]{3}{*}{ Taraxacum officinale } & polen & Muscidae semillas & \\
\hline & & Weber Syrphidae & siembras jóvenes \\
\hline & & Vespidae & \\
\hline \multirow[t]{3}{*}{ Synedrella nodiflora $L$. } & polen & Cicadellidae & semillas \\
\hline & savia & Curculionidae & orillas de caminos \\
\hline & & Vespidae & \\
\hline \multicolumn{4}{|l|}{ CUCURBITACEAE } \\
\hline \multirow[t]{3}{*}{ Cucumis sp. } & polen & Cicadellidae & semillas \\
\hline & savia & $\begin{array}{l}\text { Coreidae } \\
\text { otros }\end{array}$ & orillas de caminos \\
\hline & polen & Asilidae & semillas \\
\hline \multicolumn{4}{|l|}{ Chamaesyce } \\
\hline \multirow[t]{4}{*}{ gossypiifolia Small } & & Cicadellidae & sitios soleados \\
\hline & & Ichneumonidae & \\
\hline & & Syrphidae & \\
\hline & & Vespidae & \\
\hline
\end{tabular}


Continuación Cuadro 1

\begin{tabular}{|c|c|c|c|}
\hline Especie vegetal & Recurso explotado* & Familias insectos** & Observación*** \\
\hline $\begin{array}{l}\text { Chamaesyce hirta } \\
\text { Millspaugh }\end{array}$ & polen & $\begin{array}{l}\text { Coreidae } \\
\text { Muscidae } \\
\text { Syrphidae } \\
\text { Tachinidae }\end{array}$ & $\begin{array}{l}\text { semillas } \\
\text { en suelos desnudos }\end{array}$ \\
\hline Phyllanthus niruri $L$. & polen & $\begin{array}{l}\text { Chalcididae } \\
\text { Cicadellidae } \\
\text { Vespidae }\end{array}$ & $\begin{array}{l}\text { semillas } \\
\text { en suelos desnudos }\end{array}$ \\
\hline \multicolumn{4}{|l|}{ LAMIACEAE } \\
\hline Hyptis sp. & polen & $\begin{array}{l}\text { Braconidae } \\
\text { Coreidae } \\
\text { Eurytomidae } \\
\text { Syrphidae } \\
\text { otras }\end{array}$ & $\begin{array}{l}\text { semillas } \\
\text { en suelos desnudos }\end{array}$ \\
\hline \multicolumn{4}{|l|}{ PORTULACACEAE } \\
\hline Portulaca oleracea L. & polen & $\begin{array}{l}\text { Chrysididae } \\
\text { Vespidae }\end{array}$ & $\begin{array}{l}\text { semillas } \\
\text { en suelos desnudos }\end{array}$ \\
\hline \multicolumn{4}{|l|}{ RUBIACEAE } \\
\hline $\begin{array}{l}\text { Borreria oocimoides } \\
\text { (Burm.) DC }\end{array}$ & polen & $\begin{array}{l}\text { Braconidae } \\
\text { Chalcididae } \\
\text { Chrysomelidae } \\
\text { Phymatidae }\end{array}$ & $\begin{array}{l}\text { semillas } \\
\text { orillas de caminos }\end{array}$ \\
\hline Richardia scabra L. & polen & $\begin{array}{l}\text { Braconidae } \\
\text { Ichneumonidae } \\
\text { Muscidae } \\
\text { Tachinidae }\end{array}$ & $\begin{array}{l}\text { semillas } \\
\text { siembras jóvenes }\end{array}$ \\
\hline \multicolumn{4}{|l|}{ SOLANACEAE } \\
\hline Solanum americanum & polen & Chalcididae & semillas \\
\hline Miller & sc. glánd. & $\begin{array}{l}\text { Ichneumonidae } \\
\text { Syrphidae } \\
\text { Vespidae }\end{array}$ & suelos desnudos \\
\hline Solanum jamaicense & polen & Chalcididae & estolones \\
\hline Millspaugh & sc. glánd. & $\begin{array}{l}\text { Braconidae } \\
\text { Chrysomelidae } \\
\text { Sepsidae } \\
\text { otras }\end{array}$ & orillas de caminos \\
\hline Physalis angulata $\mathrm{L}$. & $\begin{array}{l}\text { follaje } \\
\text { sc. glánd. }\end{array}$ & $\begin{array}{l}\text { Chalcididae } \\
\text { Chrysomelidae } \\
\text { Pteromalidae }\end{array}$ & $\begin{array}{l}\text { semillas } \\
\text { orillas de caminos }\end{array}$ \\
\hline Stachystarpheta sp. & $\begin{array}{l}\text { polen } \\
\text { savia }\end{array}$ & $\begin{array}{l}\text { Cicadellidae } \\
\text { Hesperiidae } \\
\text { Nymphalidae }\end{array}$ & $\begin{array}{l}\text { semillas } \\
\text { orillas de caminos }\end{array}$ \\
\hline Verbena litoralis L. & polen & $\begin{array}{l}\text { Coreidae } \\
\text { Syrphidae } \\
\text { Tettigoniidae }\end{array}$ & $\begin{array}{l}\text { semillas } \\
\text { orillas de caminos }\end{array}$ \\
\hline
\end{tabular}


Continuación Cuadro 1

\begin{tabular}{|c|c|c|c|}
\hline Especie vegetal & Recurso explotado* & Familias insectos** & Observación $* * *$ \\
\hline \multicolumn{4}{|l|}{ PERENNES } \\
\hline Melanthera aspera & sc. glánd. & Braconidae & estolones, semillas \\
\hline Small & re. fug. & $\begin{array}{l}\text { Chalcididae } \\
\text { Eulophidae } \\
\text { Evaniidae } \\
\text { Pentatomidae } \\
\text { Pteromalidae }\end{array}$ & orillas de caminos \\
\hline $\begin{array}{l}\text { CONVOLVULACEAE } \\
\text { Ipomoea spp. }\end{array}$ & $\begin{array}{l}\text { follaje } \\
\text { re. fug. }\end{array}$ & $\begin{array}{l}\text { Chrysomelidae } \\
\text { Noctuidae } \\
\text { otras }\end{array}$ & $\begin{array}{l}\text { estolones } \\
\text { orillas de caminos y } \\
\text { de canales }\end{array}$ \\
\hline \multicolumn{4}{|l|}{ COSTACEAE } \\
\hline Costus sp. & $\begin{array}{l}\text { follaje } \\
\text { sc. glánd. }\end{array}$ & $\begin{array}{l}\text { Chrysomelidae } \\
\text { Noctuidae } \\
\text { otras }\end{array}$ & $\begin{array}{l}\text { rizomas } \\
\text { orillas de canales }\end{array}$ \\
\hline \multicolumn{4}{|l|}{ CUCURBITACEAE } \\
\hline Luffa cylindrica $\mathrm{L}$. & $\begin{array}{l}\text { follaje } \\
\text { re. fug. }\end{array}$ & $\begin{array}{l}\text { Chrysomelidae } \\
\text { Coreidae } \\
\text { Noctuidae }\end{array}$ & $\begin{array}{l}\text { semillas } \\
\text { orillas de caminos }\end{array}$ \\
\hline Momordica charantia L. & $\begin{array}{l}\text { polen } \\
\text { savia }\end{array}$ & $\begin{array}{l}\text { Coreidae } \\
\text { Pentatomidae } \\
\text { Vespidae } \\
\text { otras }\end{array}$ & $\begin{array}{l}\text { semillas } \\
\text { orillas de canales }\end{array}$ \\
\hline \multicolumn{4}{|l|}{ CYPERACEAE } \\
\hline Cyperus spp. & polen & $\begin{array}{l}\text { Braconidae } \\
\text { Ichneumonidae } \\
\text { Muscidae } \\
\text { Vespidae }\end{array}$ & $\begin{array}{l}\text { semillas } \\
\text { orillas de caminos }\end{array}$ \\
\hline Scleria melaleuca & sc. gland. & $\begin{array}{l}\text { Braconidae } \\
\text { Schlecht. y Cham. } \\
\text { Chalcididae } \\
\text { Ichneumonidae } \\
\text { Vespidae }\end{array}$ & $\begin{array}{l}\text { semillas } \\
\text { plantaciones viejas }\end{array}$ \\
\hline \multicolumn{4}{|l|}{ EUPHORBIACEAE } \\
\hline Acalipha sp. & $\begin{array}{l}\text { polen } \\
\text { follaje }\end{array}$ & $\begin{array}{l}\text { Chrysomelidae } \\
\text { Noctuidae } \\
\text { Tettigoniidae } \\
\text { Vespidae }\end{array}$ & $\begin{array}{l}\text { semillas } \\
\text { orillas de canales }\end{array}$ \\
\hline $\begin{array}{l}\text { HELICONIACEAE } \\
\text { Heliconia } \text { spp. }\end{array}$ & $\begin{array}{l}\text { follaje } \\
\text { re. fug. }\end{array}$ & $\begin{array}{l}\text { Chrysomelidae } \\
\text { Curculionidae } \\
\text { Limacodidae } \\
\end{array}$ & $\begin{array}{l}\text { rizomas } \\
\text { orillas de canales }\end{array}$ \\
\hline
\end{tabular}


Continuación Cuadro 1

\begin{tabular}{|c|c|c|c|}
\hline Especie vegetal & Recurso explotado* & Familias insectos** & Observación*** \\
\hline & & $\begin{array}{l}\text { Megalopygidae } \\
\text { otras. }\end{array}$ & \\
\hline \multicolumn{4}{|l|}{ HELICONIACEAE } \\
\hline \multirow[t]{4}{*}{ Heliconia spp. } & follaje & Chrysomelidae & rizomas \\
\hline & re. flug. & Curculionidae & orillas de canales \\
\hline & & Limacodidae & \\
\hline & & $\begin{array}{l}\text { Megalopygidae } \\
\text { otras. }\end{array}$ & \\
\hline \multicolumn{4}{|l|}{ LEGUMINOSAE } \\
\hline \multirow[t]{3}{*}{ Cajanus bicolor $\mathrm{L}}$. & polen & Chrysomelidae & semillas \\
\hline & & Curculionidae & orillas de canales \\
\hline & & Chalcididae & \\
\hline Cassia laevigata & polen & Braconidae & semillas, estacas \\
\hline \multirow[t]{3}{*}{ Willdenow } & sc. glánd. & Chalcididae & orillas de canales; \\
\hline & & Curculionidae & espacios vacantes \\
\hline & & Pentatomidae & plantación vieja. \\
\hline \multirow[t]{3}{*}{ Cassia petallaria Willd. } & polen & Braconidae & semillas \\
\hline & savia & Cicadellidae & orillas de caminos \\
\hline & & Curculionidae & \\
\hline \multirow[t]{4}{*}{ Cassia reticulata $\mathrm{L}$. } & follaje & Braconidae & semillas, estacas \\
\hline & sc. glánd. & Chalcididae & orillas de canales y \\
\hline & & Eulophidae & espacios vacantes. \\
\hline & & Formicidae & \\
\hline Senna stenocarpoides & polen & Chalcididae & semillas \\
\hline \multirow[t]{6}{*}{ (Standley) Britton } & re. fug. & Cicadellidae & orillas de caminos \\
\hline & savia & Curculionidae & \\
\hline & sc. glánd. & Evaniidae & \\
\hline & & Otitidae & \\
\hline & & Pentatomidae & \\
\hline & & Vespidae & \\
\hline \multirow[t]{5}{*}{ Crotalaria mucronata L. } & sc. glánd. & Braconidae & semillas \\
\hline & re. fug. & Chalcididae & orillas de caminos \\
\hline & & Ichneumonidae & \\
\hline & & Muscidae & \\
\hline & & Vespidae & \\
\hline \multirow{5}{*}{$\begin{array}{l}\text { Desmodium } \\
\text { ovaliofolium Waller }\end{array}$} & follaje & Chrysomelidae & semillas, estolones \\
\hline & re. fug. & Curculionidae & como cobertura \\
\hline & & Otitidae & vegetal \\
\hline & & Pentatomidae & \\
\hline & & Vespidae & \\
\hline \multirow{5}{*}{$\begin{array}{l}\text { Flemingia macrophylla } \\
\text { Willd. }\end{array}$} & polen & Chrysomelidae & semillas \\
\hline & re. fug. & Curculionidae & orillas de canales \\
\hline & & Formicidae & espacios vacantes \\
\hline & & Ichneumonidae & \\
\hline & & Vespidae & \\
\hline
\end{tabular}


Continuación Cuadro 1

\begin{tabular}{|c|c|c|c|}
\hline Especie vegetal & Recurso explotado* & Familias insectos** & Observación*** \\
\hline Centrosema pubescens & follaje & Cicadellidae & semillas \\
\hline Bentham & $\begin{array}{l}\text { re. fug. } \\
\text { savia }\end{array}$ & $\begin{array}{l}\text { Curculionidae } \\
\text { Formicidae } \\
\text { Tettigoniidae }\end{array}$ & cobertura vegetal \\
\hline Pueraria phaseoloides & follaje & Braconidae & semillas \\
\hline (Roxb.) Bentham & $\begin{array}{l}\text { re. fug. } \\
\text { savia }\end{array}$ & $\begin{array}{l}\text { Chalcididae } \\
\text { Chrysomelidae } \\
\text { Pentatomidae } \\
\text { Phymatidae } \\
\text { Vespidae } \\
\text { otras. }\end{array}$ & cobertura vegetal \\
\hline \multicolumn{4}{|l|}{ MALVACEAE } \\
\hline Hibiscus sp. & $\begin{array}{l}\text { polen } \\
\text { sc. glánd. }\end{array}$ & $\begin{array}{l}\text { Braconidae } \\
\text { Chalcididae }\end{array}$ & $\begin{array}{l}\text { semillas } \\
\text { orillas de caminos. }\end{array}$ \\
\hline Malva sp. & follaje & $\begin{array}{l}\text { Chrysomelidae } \\
\text { Curculionidae } \\
\text { otras. }\end{array}$ & $\begin{array}{l}\text { semillas } \\
\text { orillas de canales }\end{array}$ \\
\hline Sida rhombifolia L. & $\begin{array}{l}\text { polen } \\
\text { savia } \\
\text { sc. glánd. }\end{array}$ & $\begin{array}{l}\text { Lygaeidae } \\
\text { Muscidae } \\
\text { Chalcididae }\end{array}$ & $\begin{array}{l}\text { semillas } \\
\text { orillas de caminos }\end{array}$ \\
\hline Urena lobata $\mathrm{L}$. & $\begin{array}{l}\text { sc. glánd } \\
\text { polen }\end{array}$ & $\begin{array}{l}\text { Apidae } \\
\text { Chalcididae } \\
\text { Muscidae } \\
\text { Syrphidae } \\
\text { Tachinidae } \\
\text { Vespidae }\end{array}$ & $\begin{array}{l}\text { semillas, estacas } \\
\text { orillas de canales y } \\
\text { espacios vacantes }\end{array}$ \\
\hline \multicolumn{4}{|l|}{ MARANTHACEAE } \\
\hline Calathea insignis & follaje & Limacodidae & rizomas \\
\hline Petersen & re. fug. & $\begin{array}{l}\text { Megalopygidae } \\
\text { Noctuidae } \\
\text { otras. }\end{array}$ & $\begin{array}{l}\text { espacios vacantes } \\
\text { plantación vieja }\end{array}$ \\
\hline \multicolumn{4}{|l|}{ PIPERACEAE } \\
\hline Piper spp. & $\begin{array}{l}\text { follaje } \\
\text { savia }\end{array}$ & $\begin{array}{l}\text { Cercopidae } \\
\text { Curculionidae } \\
\text { Noctuidae } \\
\text { Reduviidae }\end{array}$ & $\begin{array}{l}\text { semillas } \\
\text { espacios vacantes } \\
\text { plantación adulta }\end{array}$ \\
\hline Pothomorphe peltata $\mathrm{L}$. & follaje & $\begin{array}{l}\text { Chrysomelidae } \\
\text { Curculionidae }\end{array}$ & $\begin{array}{l}\text { semillas } \\
\text { espacios vacantes }\end{array}$ \\
\hline \multicolumn{4}{|l|}{ RUBIACEAE } \\
\hline Geophila repens $\mathrm{L}$. & $\mathrm{fr}$ & $\begin{array}{l}\text { Braconidae } \\
\text { Eurytomidae }\end{array}$ & $\begin{array}{l}\text { semillas, estolones } \\
\text { en suelos desnudos } \\
\text { sitios umbríos }\end{array}$ \\
\hline Hamelia patens L. & $\begin{array}{l}\text { follaje } \\
\text { polen }\end{array}$ & $\begin{array}{l}\text { Apidae } \\
\text { Chrysomelidae } \\
\text { Vespidae }\end{array}$ & $\begin{array}{l}\text { semillas } \\
\text { orillas de caminos }\end{array}$ \\
\hline
\end{tabular}


Continuación Cuadro 1

\begin{tabular}{|c|c|c|c|}
\hline Especie vegetal & Recurso explotado* & Familias insectos** & Observación $* * *$ \\
\hline \multicolumn{4}{|l|}{ STERCULIACEAE } \\
\hline Byttneria aculeata & fo & Braconidae & semillas, estolones \\
\hline \multirow[t]{3}{*}{ Jacquin } & re & Chalcididae & orillas de canales ; \\
\hline & $\mathrm{sc}$ & Eulophidae & plantación vieja \\
\hline & & Pteromalidae & \\
\hline \multicolumn{4}{|l|}{ TILIACEAE } \\
\hline \multirow[t]{4}{*}{ Triunfetta semitriloba L. } & po & Apidae & semillas, estacas \\
\hline & re & Braconidae & orillas de caminos; \\
\hline & $\mathrm{sa}$ & Chalcididae & espacios vacantes \\
\hline & & Ichneumonidae & plantación adulta \\
\hline \multicolumn{4}{|l|}{ VERBENACEAE } \\
\hline \multirow[t]{3}{*}{ Lantana camara $\mathrm{L}$. } & fo & Cicadellidae & semillas \\
\hline & sa & Coreidae & orillas de caminos \\
\hline & & $\begin{array}{l}\text { Membracidae } \\
\text { otras. }\end{array}$ & \\
\hline \multirow[t]{2}{*}{ Lantana trifolia $\mathrm{L}}$. & fo & Cicadellidae & semillas \\
\hline & & Curculionidae & orillas de caminos \\
\hline \multicolumn{4}{|l|}{ VITIFERAE } \\
\hline \multirow[t]{8}{*}{ Cissus sycioides L. } & fo & Braconidae & semillas, estolones \\
\hline & po & Chalcididae & orillas de caminos \\
\hline & re & Ichneumonidae & \\
\hline & & Muscidae & \\
\hline & & Pteromalidae & \\
\hline & & Syrphidae & \\
\hline & & Vespidae & \\
\hline & & otras. & \\
\hline
\end{tabular}

* Recurso: $\mathrm{fo}=$ follaje; $\mathrm{fr}=$ frutos; $\mathrm{po}=$ polen; $\mathrm{re}=$ refugio; $\mathrm{sa}=\mathrm{savia} ; \mathrm{sc}=$ secreciones de glándulas extraflorales ubicadas en la lámina foliar o en los pecíolos.

** Sólo se citan algunas de làs familias de insectos más comunes.

*** Corresponde a los sitios de la plantación donde crecen o se podrían sembrar.

América tropical (Genty, et al., 1978; Reyes y Cruz, 1986; Avila y Villanueva, 1987; Mexzón , 1994; Mexzón, et al. 1996). documentó ampliamente la entomofauna de crisomélidos asociados a Zingeberales y cita a especies de Arescus, Cephaloleia y Chelobasis en Calathea y Heliconia, siendo Chelobasis perplexa Valy la especie más común en estas plantas en Centroamérica.

\section{Insectos}

La información sobre la abundancia relativa y composición de la entomofauna asociada a estas plantas ya fue presentada en otros anteriores estudios (Mexzón, 1992; 1994).
Una mayoría de los insectos perjudiciales en estas plantaciones son formas inmaduras de lepidópteros defoliadores, siendo los más importantes Euprosterna elaea Dyar, Natada pos. michorta Dyar, Oiketicus kirbyi Guilding, Opsiphanes cassina F. , Sibine megasomoides Walker (= Acharia hyperoche Dognin) y Stenoma cecropia Meyrick (Mexzón y Chinchi1la, 1992). Los estados inmaduros de esas especies rara vez alcanzan a completar su desarrollo en las siembras nuevas de palma, debido a que la fauna entomófaga es abundante. Por 10 contrario, en las plantaciones viejas sí ocurren pérdidas económicas causadas por insectos defoliadores, debido a la escasez de enemigos naturales (Genty, 1989; Mexzón, 1994). 
Cuadro 2. Especies vegetales en las que se alimentan insectos defoliadores de la palma aceitera en el Pacífico Sur de Costa Rica (1990-92).

\begin{tabular}{ll}
\hline Especie vegetal & \multicolumn{1}{c}{ Especie insectil } \\
\hline Heliconia spp. & Euclea sp. inmad/adult \\
& Euclea diversa Druce inmad/adult \\
& Megalopyge sp.inmad/adult \\
& Mesocia dumilla Dyar inmad/adult \\
& Natada pos. michorta Dyar inmad/adult \\
& Oiketicus kirbyi Guilding inmad/adult \\
& Saliana severus Mabille inmad/adult \\
& Spaethiella pos. costipennis Bohemanninmad/adult \\
& Acharia apicalis Dyar inmad/adult \\
& Acharia hyperoche Dognin inmad/adult \\
& Brassolis sp. inmad/adult \\
Calathea insignis Petersen & Phobetron sp. inmad/adult \\
& Peleopoda arcanella Busck inmad/adult \\
Cassia reticulata L. & O. kirbyi inmad/adult \\
& A. hyperoche inmad/adult \\
\hline
\end{tabular}

Algunos insectos benéficos residen en la vegetación del piso de la plantación (Delvare y Genty, 1992; Mexzón, 1992; Mexzón y Chinchilla, 1992); entre ellos los principales fueron chinches pentatómidos y avispas parasitoides (Cuadro 3). Los chinches Alcaeorrhynchus grandis (Dallas) y Podisus sp. fueron comunes en el kudzú donde depredaban las larvas de Anticarsia gemmatalis (Hubner) y de Estigmene acrea Druce que son muy comunes en este medio.

Durante los incrementos poblacionales de A. hyperoche, $O$. cassina y $S$. cecropia se observaron ninfas y adultos de los chinches pentatómidos A. grandis, Mormidea sp. y Podisus spp. depredando gran cantidad de larvas de estas mariposas.

Estos residían en la vegetación circundante e incrementaron rápidamente su población en respuesta a la abundancia del recurso alimentario.

Varias especies de los géneros Brachymeria, Сопиra, Cotesia, Digonogastra, Horismenus y otros se observaron alimentándose en la vegetación. Delvare y Genty (1992) encontraron a estas avispas asociadas a malezas Asteraceae, Cyperaceae, Euphorbiaceae, Malvaceae, Solanaceae y Verbenaceae en plantaciones de Colombia y Ecuador. Este resultado de los autores mencionados es similar a los obtenidos en Costa Rica (Mexzón y Chinchilla, 1 990a; 1990b; 1992; Mexzón, 1992).

Para que haya una permanencia de los artrópodos entomófagos en la plantación, se deben reunir condiciones adecuadas para su establecimiento como la provisión de recursos alimentarios, sitios de refugio y condi- ciones de microclima adecuadas. Estas condiciones podrían ser reunidas por áreas de vegetación poco perturbadas donde las poblaciones se puedan estabilizar en el tiempo.

\section{Pautas para el manejo de la vegetación}

El incremento de la diversidad estructural del agroecosistema puede ser logrado por el crecimiento de las malezas en el monocultivo.

Los resultados obtenidos en cultivos con malezas demuestran que la diversificación vegetal disminuyó los problemas con insectos fitófagos (Risch et al., 1983). El concepto de manejo de la vegetación en un cultivo como la palma aceitera requiere la evolución del esquema del combate químico a uno de manejo racional de la vegetación; esto significa para el palmicultor 1- una menor inversión económica en la aplicación de productos y en el combate mecanizado de las malezas y 2- la conservación de un conjunto de factores bióticos que son necesarios para las poblaciones de organismos residentes.

Los espacios donde puedan crecer las malezas como, por ejemplo, franjas de terreno a lo largo de los caminos y de los canales de drenaje primarios podrían funcionar como "corredores biológicos" por los cuales se puedan movilizar los insectos hacia el interior de la plantación. También la siembra con malezas específicas de los espacios que quedaron vacantes por la muerte palmas podrían funcionar como "islas biológicas" en las cuales se refugien y alimenten los insectos. 
Cuadro 3. Enemigos naturales de plagas de la palma aceitera y algunas de las especies vegetales huéspedes encontradas en el Pacífico Sur de Costa Rica(1990-92).

\begin{tabular}{|c|c|c|}
\hline Enemigo natural & Presa/Huesped & Especie vegetal \\
\hline \multicolumn{3}{|l|}{ HEMIP PÉNTATOMIDAE } \\
\hline \multirow{3}{*}{ Alcaeorrynchus grandis } & Automeris sp. & Cissus sycioides \\
\hline & Opsiphanes cassina & Pueraria phaseoloides \\
\hline & Stenoma cecropia & \\
\hline \multirow[t]{4}{*}{ Podisus spp. } & O. cassina & C. sycioides \\
\hline & Acharia spp. & Melanthera aspera \\
\hline & Talima straminea & P. phaseoloides \\
\hline & S. cecropia & \\
\hline \multirow[t]{4}{*}{ Especie sin identificar } & Euclea sp. & Borreria sp. \\
\hline & O. cassina & C. sycioides \\
\hline & A. ophelians & M. aspera \\
\hline & $\cdot$ & P. phaseoloides \\
\hline \multicolumn{3}{|l|}{ HYM.:BRACONIDAE } \\
\hline \multirow[t]{4}{*}{ Cotesia spp. } & Acharia sp. & Ageratum conyzoides \\
\hline & Acharia hyperoche & Amarantus spinosus \\
\hline & Acharia ophelians & Borreria sp. \\
\hline & & Triunfetta semitriloba \\
\hline \multirow[t]{3}{*}{ Digonogastra diversus } & Oiketicus kirbyi & A. spinosus \\
\hline & & Flemingia macrophylla \\
\hline & & Scleria melaleuca \\
\hline \multirow[t]{3}{*}{ Microgastrinae no identificado } & Acharia sp. & Solanum jamaicense \\
\hline & A. ophelians & T. semitriloba \\
\hline & Euprosterna elaea & A. spinosus \\
\hline \multicolumn{2}{|l|}{ HYM.: CHALCIDIDAE } & Baltimora recta \\
\hline \multirow[t]{2}{*}{ Conura spp. } & A. hyperoche & Crotalaria mucronata \\
\hline & A. ophelians & S. melaleuca \\
\hline Conura spp & O. kirbyi & Flemingia macrophylla \\
\hline Conura oiketicussi & O. kirbyi & S. jamaicense \\
\hline Conura maculata & o. Cassina & A.spinosus \\
\hline Conura inmaculata & O. cassina & B. recta \\
\hline Brachymeria sp. & A. hyperoche & M. aspera \\
\hline \multicolumn{3}{|l|}{ HYMEN.: EULOPHIDAE } \\
\hline Horismenus sp. & O. cassina & M. aspera \\
\hline \multirow{2}{*}{ Trichospilus diatrae } & Peleopoda arcanella & Cassia reticulata \\
\hline & S. cecropia & M. aspera \\
\hline \multicolumn{3}{|l|}{ HYMEN.: ENCYRTHIDAE } \\
\hline \multirow[t]{2}{*}{ Ooencyrtus sp. } & Automeris sp. & Byttneria aculeata \\
\hline & O. cassina & S. melaleuca \\
\hline \multicolumn{3}{|l|}{ HYMEN.: ICHNEUMONIDAE } \\
\hline \multirow[t]{4}{*}{ Casinaria $s p$} & Acharia spp. & A. spinosus \\
\hline & Euprosterna elaea & Chamaesyce gossypiifolia \\
\hline & Megalopyge sp. & Solanum americanum \\
\hline & Natada pos. michorta & \\
\hline \multicolumn{3}{|l|}{ HYMEN.: SCELIONIDAE } \\
\hline \multirow[t]{2}{*}{ Telenomus sp. } & Automeris sp. & B. aculeata \\
\hline & O. cassina & M. aspera \\
\hline
\end{tabular}


Janzen (1968) consideró las plantas como islas en el espacio y en el tiempo para los insectos y también se podrían visual izar áreas con malezas dentro de las plantaciones como islas, donde los insectos se movilicen, alimenten o refugien.

El tamaño y número de islas por unidad de área que son necesarios para obtener un buen control natural de los insectos perjudiciales es difícil de decir sin una prueba experimental con diferentes tamaños y números de islas por hectárea, sin embargo en las plantaciones viejas los espacios vacantes pueden ser utilizados como sitios de refugio para los insectos. En la práctica consistiría sólo una sustitución de las gramíneas que crecen en algunos de estos espacios, por malezas de hoja ancha con gran capacidad de atracción de insectos.

Es opinión del autor, que cuatro islas de 100 metros cuadrados $(10 \times 10 \mathrm{~m})$ por hectárea, serían adecuadas para sostener la entomofauna dentro del cultivo. En áreas con estas dimensiones se podría tener una alta tasa de inmigración de los insectos desde los bordes del cultivo, una baja tasa de emigración y posibilidades de sobrevivencia y reproducción.

Los corredores e islas, se pueden hacer a través de prácticas culturales como: 1) la selección cuidadosa y fomento de especies vegetales de interés, 2) el combate químico y mecánico de las malezas en forma selectiva, 3) la recolección de semillas y su dispersión en sitios específicos de la plantación o la siembra de partes vegetativas de algunas especies que son fáciles de propagar en forma asexual (por medio de estacas, estolones, rizamas, etc.); 4) el establecimiento de viveros de las especies promisorias con el propósito de distribuirIas en las plantaciones con mayor facilidad. En este programa es fundamental 5) el entrenamiento del personal agrícola en el manejo de estas especies y 6) su capacitación a través de charlas, de material didáctico como una guía de campo con fotografías a color, de murales de fotografías y de hojas técnicas de divulgación.

La inversión inicial para el establecimiento de los corredores e islas, la instalación de los viveros de malezas y para el programa de capacitación del trabajador agrícola, es gradual y se realiza en un período de varios años; sin embargo, esta diversificación puede promover grandes beneficios a mediano plazo que son cuantificables en un menor gasto en herbicidas y combate mecánico de las malezas y otros que no son cuantificables como la conservación de los suelos, el incremento de las poblaciones de enemigos naturales, un mayor número de microclimas (Risch, et al., 1983; Letourneau y Altieri 1983), un mejor control de las malezas indeseables a través del uso de las coberturas de leguminosas y una serie de condiciones bióticas no consideradas aquí.
Finalmente, se recalca la necesidad de apoyar su uso en una actitud responsable y en estudios ecológicos que permitan la evaluación del efecto de estas medidas sobre las poblaciones de insectos y sobre la salud y producción del cultivo para poder hacer los cambios que sean necesarios, de forma que la relación beneficio/costo sea favorable. Además para evitar que un posible agente patogénico a la palma sea favorecido.

Estas medidas podrían ser aplicadas a otros cultivos frutales perennes que no posean un insecto plaga muy polífago y se deben de realizar con especies vegetales propias de la región.

Este es un proceso gradual que no se puede implementar en corto tiempo; los resultados obtenidos en esta región y en este cultivo no son necesariamente verdaderos para otras regiones y para otros cultivosd hasta tanto no existan los estudios de campo que apoyen los criterios a tomar.

\section{AGRADECIMIENTO}

El autor agradece al Dr. C.M. Chinchilla de Palma Tica S.A./ASD de Costa Rica por las facilidades para realizar este estudio; a G. Castrillo por su valiosa ayuda en el campo y al Dr. P.E. Hanson de la Escuela de Biología de la Universidad de Costa Rica por la identificación taxonómica de las avispas parasitoides.

\section{LITERATURA CITADA}

ALTIERI, M.A. 1983. Agroecología. Bases científicas de la agricultura alternativa. Valparaiso, Chile. Centro de Estudios Apropiados para América Latina. 184 p.

ÁVILA, M.; VILLANUEVA, A. 1987. El gusano canasta, Oiketicus kirbyi Guilding. Boletín Técnico FEDEP ALMA (Colombia) No 2. 28 p.

BOMBOSCH, S. 1973. Occurrence of enemies on different weeds with aphids. In: Ecology of aphidophagous insects. Hodek, Y., ed. Academia Pub. House, Prague. pp. 177-179.

BRAKEN , G.K. 1969. Effect of dietary amino acids, salts and starvation on the fecundity of the parasitoid Exeristes comstockii (Hymenoptera: Ichneumonidae). Canadian Entomologist 101: 91-96.

CHINCHILLA, C. 1992. Fauna perjudicial y enfermedades de la palma aceitera. ASD de Costa Rica, San José, Costa Rica. 134 p. 
DELVARE, G.; GENTY, P. 1992. Interés de las plantas atractivas para la fauna auxiliar de las plantaciones de palma aceitera en América tropical. Oléagineux 47(10):551558.

GENTY, P. 1984. Estudios entomológicos con relación a la palma aceitera en América tropical. Palmas (Colombia) 5 (1): 22-31.

GENTY, P. 1989. Manejo y control de plagas de la palma aceitera en América tropical. Curso ASD para agrónomos y administradores de Palmas del Oriente, Colombia. 11 p. (mimeografiado).

GENTY,P.; DESMIER DE CHENON, R; MORIN,J.P. 1978. Plagas de la palma africana en América Latina. Oléagineux (número especial) 33 (7):324-420.

HAGEN, D.S. 1976. Role ofnutrition in insect management. Proc. Tall Timbers Conference on Ecological Animal Control by Habitat Management 6:261-262.

HOONG; H.W.; HOH CRISTOPHER, KY. 1992. Major pests of oil palm in Sabah.The Planter (Malaysia) 68 (793): 193-21 O.

JANZEN, D.H. 1968. Host Plant as Islands in Evolutionary and Contemporary Time. American Naturalist 102:592595.

LEIUS, K. 1967. Food sources and preferences of adults of a parasite, Scambus buolianae (Hym.: Ichneumonidae) and their consequences. Canadian Entomology 99:865887.

LETOURNEAU, D.K.; ALTIERI, M.A. 1983. Abundance patterns of, predator orius tristicolor (Hemiptera: Anthocoridae) and Its Prey, Frankliniella occidentalis (Thysanoptera:Thripidae): Habitat Attraction in Polycultures versus Monocultures.Environ. Entomol. 12: 1464-1469.

MEXZON, RG. 1992. Insectos visitantes de malezas: manejo y conservación de la vegetación para incrementar los enemigos naturales de plagas de la palma aceitera (Elaeis guineensis Jacquin). ler. Congr. Centroamericano de Entomología y Combate Natural de Plagas. San José, Costa Rica. 14p.

MEXZON, R.G. 1994. Algunas pautas de manejo de las malezas para incrementar los enemigos naturales en el cultivo de la palma aceitera (Elaeis guineensis Jacquin).V
Congreso Internacional de Manejo Integrado de Plagas. San José, Costa Rica.

MEXZON, RG.; CHINCHILLA, C.M. 1990a. Las interacciones entre insectos parasitoides con malezas en un agroecosistema de palma de aceite (Elaeis guineensis Jacquin, Palmae) en América tropical. IV Congr. Nal. y III Congr. Intl.de MIP, Managua, Nicaragua.

MEXZON, R.G.; CHINCHILLA, C.M. 1 990b. Reconocimiento de la entomofauna perjudicial, enemigos naturales y malezas útiles en el cultivo de la palma aceitera (Elaeis guineensis Jacquin) en América Central. 1 Congr. Nal. de Entomología, Universidad Nacional, Heredia, Costa Rica. 7 p.

MEXZON, RG.; CHINCHILLA, C.M. 1992. Entomofauna petjudicial, enemigos naturales y malezas útiles en palma aceitera en América Central. Manejo Integrado de Plagas (C.R) 20/21:1-7.

MEXZON, RG.; CHINCHILLA, C.M.; SALAMANCA, D.E. 1996. Biología de Sibine megasomoides Walker (Lepidoptera:Limacodidae): observaciones de la plaga en el cultivo de la palma aceitera en Parrita, Costa Rica. ASD Oil Palm Papers (Costa Rica) No 12: 1-10.

REYES, A.; CRUZ, M.A. 1986. Principales plagas de la palma de aceite (Elaeis guineensis Jacq.) en América tropical: su manejo y su control. Curso de entrenamiento. United Brands Co., Quepas, Costa Rica. 55p.

RISCH, S.1.; ANDOW, D.; ALTIERI, M.A. 1983. Agroecosystem diversity and pest control: data, tentative conclusions and new research directions. Environmental Entomology 12:625-629.

ROOT, RB. 1973. Organization of a plant-arthropod association in simple and diverse habitats: the fauna of collards (Brassica oleracea). Ecological Monographs 43(1): 95124.

SYED, R.A.; SHAH, J. 1976. Some important aspects ofinsect pest management in oil palm estates in Sabah, Malaysia. In: International Developments in Oil Palm. Earp, D.A.; Newall, W., eds. The Incorporated Soco ofPlanters,Malasia. pp. 577-590.

SYME, P.D. 1975. The effects offlowers on the longevity and fecundity of two native parasites of the European shoot moth in Ontario. Environmental Entomology 4:337.340 . 